\title{
Sulphite efflux pumps in Aspergillus fumigatus and dermatophytes
}

\section{Correspondence \\ Michel Monod \\ Michel.Monod@chuv.ch}

Received 10 October 2006

Revised 23 November 2006

Accepted 7 December 2006

\author{
Barbara Léchenne, ${ }^{1}$ Utz Reichard, ${ }^{2}$ Christophe Zaugg, ${ }^{1}$ Marina Fratti, ${ }^{1}$ \\ Jiri Kunert, ${ }^{3}$ Olivier Boulat ${ }^{4}$ and Michel Monod ${ }^{1}$ \\ ${ }^{1}$ Department of Dermatology, Centre Hospitalier Universitaire Vaudois, Lausanne, Switzerland \\ ${ }^{2}$ Department of Medical Microbiology and National Reference Center for Systemic Mycoses, \\ University Hospital of Göttingen, Germany \\ ${ }^{3}$ Department of Biology, Faculty of Medicine, Olomouc, Czech Republic \\ ${ }^{4}$ Clinical Chemistry Laboratory, Centre Hospitalier Universitaire Vaudois, Lausanne, Switzerland
}

Dermatophytes and other filamentous fungi excrete sulphite as a reducing agent during keratin degradation. In the presence of sulphite, cystine in keratin is directly cleaved to cysteine and $S$ sulphocysteine, and thereby, reduced proteins become accessible to hydrolysis by a variety of secreted endo- and exoproteases. A gene encoding a sulphite transporter in Aspergillus fumigatus (AfuSSU1), and orthologues in the dermatophytes Trichophyton rubrum and Arthroderma benhamiae (TruSSU1 and AbeSSU1, respectively), were identified by functional expression in Saccharomyces cerevisiae. Like the S. cerevisiae sulphite efflux pump Ssu1p, AfuSsu1p, TruSsu1p and AbeSsu1p belong to the tellurite-resistance/dicarboxylate transporter (TDT) family which includes the Escherichia coli tellurite transporter TehAp and the Schizosaccharomyces pombe malate transporter Mae1p. Seven genes in the A. fumigatus genome encode transporters of the TDT family. However, gene disruption of AfuSSU1 and of the two more closely related paralogues revealed that only AfuSSU1 encodes a sulphite efflux pump. TruSsulp and AbeSsulp are believed to be the first members of the TDT family identified in dermatophytes. The relatively high expression of TruSSU1 and AbeSSU1 in dermatophytes compared to that of AfUSSU1 in A. fumigatus likely reflects a property of dermatophytes which renders these fungi pathogenic. Sulphite transporters could be a new target for antifungal drugs in dermatology, since proteolytic digestion of hard keratin would not be possible without prior reduction of disulphide bridges.

\section{INTRODUCTION}

The dermatophytes are a group of common closely related fungi which are responsible for the great majority of human superficial mycoses (Kwong-Chung \& Bennet, 1992; Weitzman \& Summerbell, 1995). These highly specialized pathogenic fungi are almost exclusively found in the epidermal stratum corneum, hair or nails. The battery of proteases secreted by dermatophytes is similar to that of Aspergillus, but differs by multiple endoprotease members of the S8 (subtilisins) and M36 (fungalysins) family (see MEROPS proteolytic enzyme database; http://merops.sanger. ac.uk) (Jousson et al., 2004a, b). Although it is evident that the secreted proteolytic activity of these fungi is important for degradation of compact keratinous tissues, fungal

Abbreviation: TDT, tellurite-resistance/dicarboxylate transporter.

The GenBank/EMBL/DDBJ accession numbers for the AfuSSU1, AfuSSUL1, AfuSSUL2, TruSSU1 and AbeSSU1 sequences reported in this paper are AY861352, AY861353, AY861354, DQ777768 and EF035480, respectively. secreted proteases are incapable by themselves of dissolving structures made of hard keratin which are rich in cystine. Efficient keratin degradation by hydrolytic enzymes has to be accompanied by the simultaneous reduction of cystine disulphide bridges, which are mainly responsible for the resistant nature of keratin (Kunert, 1992, 2000). Moreover, efficient degradation in vitro of the hair structure of keratin azure by hydrolytic enzymes is only possible in the presence of a reducing agent, e.g. $1 \% \beta$-mercaptoethanol or DTT (Jousson et al., 2004b).

During keratin degradation, dermatophytes and filamentous fungi have been shown to excrete sulphite as a reducing agent (Kunert, 1972a, 1976). In the presence of sulphite, disulphide bonds of the keratin substrate are directly cleaved to cysteine and $S$-sulphocysteine. The presence of sulphocysteine has been confirmed by histochemical methods in human hair attacked by Microsporum gypseum (Kunert, 1972b). This compound was found in the free form and in oligopeptides of $\sim 700-2500 \mathrm{Da}$ which were products of keratinolysis (Kunert, 1976; Ruffin et al., 1976). As highly 
specialized fungi, the dermatophytes are able to metabolize free cystine added to a nutrient broth, and excrete excess sulphur as sulphate and sulphite. Because sulphite is immediately consumed by sulphitolysis of disulphide bridges, this compound is only detectable when its amount exceeds that of cystine (Kunert, 1975, 2000).

The necessity to reduce disulphide bridges in the digestion of cornified tissues instigated our investigation of the mechanism of sulphite secretion in dermatophytes. In dermatophytes, as well as in the opportunistic mould Aspergillus fumigatus, we have identified genes encoding sulphite efflux pumps by heterologous expression in Saccharomyces cerevisiae, which results in a sulphite-resistant phenotype. Sulphite transporters in keratinolytic fungi could be a new target for antifungal drugs in dermatology.

\section{METHODS}

Strains and plasmids. Trichophyton rubrum CHUV1673-05, Arthroderma benhamiae CBS112371 (Fumeaux et al., 2004) and A. fumigatus D141 (NRRL 6585, US Department of Agriculture, Peoria, IL) were used in this study. All plasmid subcloning experiments were performed in Escherichia coli XL-1 Blue using plasmids pMTL21 (Chambers et al., 1988), pUC18 and pCL1920. The diploid S. cerevisiae strain INVSc1 (MATa, his3D1, leu2, trp1-289, ura3-52; $M A T \alpha$, his3D1, leu2, trp1-289, ura3-52) and the expression vector pYES2 (Invitrogen) were used to express heterologous transporters.

Growth media. T. rubrum, Arth. benhamiae and A. fumigatus were grown on Sabouraud agar and liquid medium (Bio-Rad), or in soy protein liquid medium (SP) and keratin liquid medium (KSP) (Monod et al., 2005) to promote production of proteolytic activity, as previously described.

Cystine-arginine liquid medium (CAM) [0.8\% (w/v) glucose, $0.2 \%$ (w/v) arginine, $0.04 \%(w / v) \mathrm{KH}_{2} \mathrm{PO}_{4}, 3 \mathrm{mM}(0.072 \%$, w/v) cystine] (Kunert, 1982) was used to demonstrate secretion of sulphite. Cystine was added to the sterile medium in the form of a $10 \times$ concentrated solution in $\mathrm{HCl}(1 \mathrm{M})$ and the medium was neutralized with $\mathrm{KOH}$. A volume of $100 \mathrm{ml}$ liquid medium was inoculated with a plug of freshly growing mycelium in $800 \mathrm{ml}$ tissue-culture flasks. The cultures were incubated for 10 days at $30^{\circ} \mathrm{C}$ without shaking.

Complete medium for S. cerevisiae was YEPD [2\% (w/v) Difco Bacto peptone, $1 \%(\mathrm{w} / \mathrm{v})$ Difco Bacto yeast extract, $2 \%(\mathrm{w} / \mathrm{v})$ glucose]. $S$. cerevisiae synthetic minimal medium supplemented with histidine, leucine and tryptophan $\left(20 \mu \mathrm{g} \mathrm{ml}^{-1}\right)$ was prepared according to Sherman (1991). For expression of genes cloned in pYES2 under the control of the GAL promoter, galactose was added instead of glucose as the carbon source. YEPD + TA (YEPD containing $75 \mathrm{mM}$ L-tartaric acid and buffered at $\mathrm{pH}$ 3.5) was prepared as described elsewhere (Park et al., 1999). YEPG + TA was prepared in the same way as YEPD + TA with $2 \%$ galactose instead of glucose. YEPG + TA plates containing sulphite were prepared by spreading an appropriate amount of freshly made $\mathrm{Na}_{2} \mathrm{SO}_{3}$ stock solution $(0.25$ or $0.5 \mathrm{M})$ onto agar plates containing $25 \mathrm{ml}$ medium, and allowing the sulphite to dry and diffuse overnight at room temperature.

Sulphite and $\boldsymbol{S}$-sulphocysteine analysis. Supernatants of cultures in SP and KSP media were first treated by Pronase $\left(10 \mu \mathrm{g} \mathrm{ml}^{-1}\right)$ (Roche Diagnostics) and porcine kidney aminopeptidase $\mathrm{M}\left(2 \mathrm{U} \mathrm{ml}^{-1}\right)$ (Calbiochem) for $24 \mathrm{~h}$ at $37^{\circ} \mathrm{C}$. Free $\mathrm{S}$-sulphocysteine was determined after deproteinization treatment of the sample with $32 \%(w / v)$ sulphosalicylic acid. Amino acid analysis was performed by HPLC with a fluorescence detector, after precolumn derivatization with $\mathrm{O}$-phthaldialdehyde-3-mercaptopropionate and 9-fluorenylmethyl-chloroformate (FMOC) (Henderson et al., 2000). The concentration of free sulphite was determined by the fuchsin-formaldehyde method (Scaringelli et al., 1967).

Expression of tellurite-resistance/dicarboxylate transporters (TDTs) in $\boldsymbol{S}$. cerevisiae. cDNAs encoding the transporters AfuSsulp, AfuSsullp, AfuSsul2p, TruSsulp and AbeSsulp were obtained by RT-PCR using a OneStep RT-PCR kit (Qiagen). Briefly, $20 \mathrm{ng}$ total RNA, $10 \mu \mathrm{l}$ of the supplied $5 \times$ OneStep RT-PCR buffer (12.5 $\mathrm{mM} \mathrm{MgCl}_{2}, \mathrm{pH} 8.7$ ), $2 \mu \mathrm{l}$ deoxynucleotide mix containing $10 \mathrm{mM}$ each dNTP, $5 \mu \mathrm{l}$ sense and antisense primers (P1-P8, Table 1) at a concentration of $6 \mu \mathrm{M}$, and $2 \mu \mathrm{l}$ OneStep RT-PCR enzyme mix were mixed on ice, and subsequently incubated at $50{ }^{\circ} \mathrm{C}$ for $30 \mathrm{~min}$ and $95^{\circ} \mathrm{C}$ for $15 \mathrm{~min}$. The reaction mixtures were then subjected to 35 cycles of $0.5 \mathrm{~min}$ at $94^{\circ} \mathrm{C}, 0.5 \mathrm{~min}$ at $55^{\circ} \mathrm{C}$ and $1 \mathrm{~min}$ at $72^{\circ} \mathrm{C}$, and finally incubated for $10 \mathrm{~min}$ at $72^{\circ} \mathrm{C}$. Alternatively, a cDNA library of A. fumigatus D141 (Denikus et al., 2005) was used to amplify cDNAs encoding A. fumigatus transporters. PCR was performed with the homologous primers P1-P6 (Table 1) and $200 \mathrm{ng}$ of DNA prepared from $10^{6}$ clones of the cDNA library, using a standard protocol (Jousson et al., 2004a, b).

A DNA fragment encoding SceSsulp was amplified by PCR using the primers P9 and P10 (Table 1), and S. cerevisiae INVSc1 genomic DNA as a target. For amplification, a standard protocol was used (Jousson et al., 2004a, b).

Expression plasmids were constructed by cloning PCR products in the $S$. cerevisiae expression vector pYES2. The PCR products were purified with a PCR purification kit (Roche Diagnostics) and then digested with restriction enzymes for which a site was previously designed at the $5^{\prime}$ end of the primers. The cloned fragments were further sequenced and the absence of possible PCR-induced errors was confirmed. Plasmid DNA was prepared from one E. coli clone harbouring a correct construct. S. cerevisiae transformations were performed using a transformation kit and according to the recommendations of the supplier (Invitrogen). Selection of URA3 transformants was performed using minimal medium with glucose and required amino acids.

Transformants were tested for sulphite resistance using low-pH medium, as described by Park et al. (1999). Yeasts were grown to the mid-exponential phase $\left(\mathrm{OD}_{600} 1.0\right)$ at $30^{\circ} \mathrm{C}$ in minimal medium with galactose and required amino acids. Each culture was diluted to $\mathrm{OD}_{600}$ 1.0. Subsequently, $5 \mu \mathrm{l}$ of serial dilutions $\left(10^{0}-10^{4}\right)$ were spotted onto YEPG + TA plates containing the desired concentration of sulphite. The plates were incubated at $30^{\circ} \mathrm{C}$ for 2 days.

A. fumigatus gene disruption. Gene disruption vectors were constructed using pAN7.1 (Punt et al., 1987) and $1.0 \mathrm{~kb}$-sized internal fragments of the respective A. fumigatus gene. In detail, gene fragments were obtained by PCR using appropriate primers (P11-P16, Table 1) and genomic A. fumigatus DNA as the template. The PCR products were first cloned into pCR-Script Amp SK(+) (Stratagene). In a second step, the A. fumigatus fragments were excised from the plasmid constructs with StuI and BstEII, for which a site was introduced into the primers, and ligated to the larger fragment of pAN7.1 digested with the same restriction enzymes. The generated plasmids were termed $\mathrm{p} \Delta S S U 1, \mathrm{p} \Delta S S U L 1$ and $\mathrm{p} \Delta S S U L 2$. Undigested plasmids were used for subsequent gene-targeted disruption experiments.

Transformation of A. fumigatus D141 was performed according to a protocol that has been used for Aspergillus nidulans and A. fumigatus (Tilburn et al., 1983; Paris, 1994) with $10^{7}$ protoplasts and $5 \mu \mathrm{g}$ of plasmid. After overnight expression of the hygromycin B phosphotransferase gene $(H P H)$, the transformants were incubated on agar 
Table 1. Primers used in this study

Nucleotides in italic type represent cloning sites.

\begin{tabular}{|c|c|c|c|}
\hline Primer & Oligonucleotide sequence & Location & $\begin{array}{l}\text { PCR product size } \\
\text { with cloning sites }\end{array}$ \\
\hline P1 & 5'-GTTAAGCTTCAACATGAGCTGCGGCAATACAAAT-3' & AfuSSU1 & $1213 \mathrm{bp}$ \\
\hline P3 & 5'-GTTGAGCTCCAACATGGAACCCCGTCTCAAACCA-3' & AfuSSUL1 & 1213 bp \\
\hline $\mathrm{P} 4$ & 5'-CTTTCTAGATCATGCTTGGCGAATTGGAGC-3' & Complement of AfuSSUL1 & $\mathrm{SacI} / \mathrm{Xba \textrm {I }}$ \\
\hline P7 & $\begin{array}{l}\text { 5'-GTTAAGCTTCAACATGCCATCAGGATCCGGCTTCCATAA- } \\
\text { CATCG-3' }\end{array}$ & TruSSU1/AbeSSU1 & $1153 \mathrm{bp} / 1153 \mathrm{bp}$ \\
\hline P8 & 5'-CTTGAATTCTCATCGGAAGAGCTCCTTGGTGCATGA-3' & Complement of TruSSU1/AbeSSU11 & HindIII/EcoRI \\
\hline P9 & 5'-GTTGAGCTCCAACATGGTTGCCAATTGGGTACTT-3' & SceSSU1 & $1399 \mathrm{bp}$ \\
\hline $\mathrm{P} 10$ & 5' -CTTGAATTCTTATGCTAAACGCGTAAAATC-3' & Complement of SceSSU1 & XhoI/EcoRI \\
\hline P15 & 5'-TAGGTGACCAGTGGCTGGAGACGAATCGTG-3' & AfuSSUL2 & $990 \mathrm{bp}$ \\
\hline P16 & 5'-TAAGGCCTCTACGACATAAACTATGGAGGCG-3' & Complement of AfuSSUL2 & BstEII/StuI \\
\hline P17 & 5'-TCCATCAGATAATGAGCTGCGG-3' & Upstream sequence in AfuSSU1 & 1153 bp \\
\hline $\mathrm{P} 18$ & 5'-GAATATCCTCTTGACACCGCTCC-3' & pAN7.1 sequence & \\
\hline P19 & 5'-GGTTAAGATCCACGTCTTCGGG-3' & pAN7.1 sequence & 1069 bp \\
\hline P20 & 5'-GAGCGATAGAAGCTGACGCG-3' & Downstream sequence in $A f u S S U 1$ & \\
\hline $\mathrm{P} 21$ & 5'-AATGGAACCCCGTCTCAAACC-3' & Upstream sequence in AfuSSUL1 & 1152 bp \\
\hline $\mathrm{P} 22$ & 5'-TATCCTCTTGACACCGCTCCG-3' & pAN7.1 sequence & \\
\hline $\mathrm{P} 23$ & 5'-GTCGATCATCCGCTGAAGGC-3' & pAN7.1 sequence & $1120 \mathrm{bp}$ \\
\hline P24 & 5'-CGGAAATGTGAATCCCCACC-3' & Downstream sequence in AfuSSUL1 & \\
\hline
\end{tabular}

based on minimal medium (Cove, 1966) containing $200 \mu \mathrm{g}$ hygromycin $\mathrm{ml}^{-1}$ (Sigma), and selected after 5 days incubation at $20^{\circ} \mathrm{C}$ followed by an overnight incubation at $42^{\circ} \mathrm{C}$. Transformants initially identified as hygromycin resistant were chosen and subcultured again on agar containing hygromycin. Typically, 100-200 hygromycinresistant colonies were obtained for each transformation using $10^{7}$ protoplasts and $5 \mu \mathrm{g}$ plasmid DNA.

The A. fumigatus disruptants were identified by PCR of genomic DNA from various numbers of hygromycin-resistant colonies as a template and two pairs of specific primers (P17-P28, Table 1). Each primer pair was designed to yield a product of a predicted size when the respective plasmid had integrated at a homologous site. In each primer pair, one primer hybridized with the transformation plasmid and the other primer hybridized with genomic DNA near the desired homologous integration locus, as exemplarily shown for the AfUSSU1 disruption (Fig. 1). The primer pairs P17 to P20, P21 to P24 and P25 to P28 (Table 1) were used for screening ssul, ssull and ssul2 mutants, respectively.

Positive PCR screening of 20 hygromycin-resistant colonies in each experiment led to the identification of three, one and 10 disruption mutants for the genes encoding AfuSsulp, AfuSsullp and AfuSsul2p, respectively. Neither the wild-type parental strain nor those transformants with presumed ectopically integrated plasmids showed a positive reaction.

Sulphite resistance of $\boldsymbol{A}$. fumigatus and dermatophytes. $A$. fumigatus and dermatophytes were grown for 4 and 10 days, respectively, at $30^{\circ} \mathrm{C}$ on Sabouraud medium. YEPD + TA plates containing the desired concentration of sulphite were inoculated with A. fumigatus conidia or dermatophyte mycelium. Alternatively, $100 \mathrm{ml}$ liquid YEPD + TA medium containing sulphite was inoculated in $800 \mathrm{ml}$ tissue-culture flasks. Dermatophyte and A. fumigatus cultures were incubated for 10 and 3 days, respectively, at $30^{\circ} \mathrm{C}$ without shaking.

T. rubrum and Arth. benhamiae gene cloning. Recombinant plaques $\left(2 \times 10^{4}\right)$ of a previously constructed T. rubrum $\lambda$ EMBL3 genomic DNA library were immobilized on GeneScreen nylon membranes (NEN Life Science Products). The filters were hybridized with a ${ }^{32} \mathrm{P}$-labelled AfUSSU1 probe under low-stringency conditions (Monod et al., 1994). All positive plaques were purified, and the associated bacteriophage DNAs were isolated as described elsewhere (Grossberger, 1987). Agarose gel electrophoresis of restricted recombinant bacteriophage $\lambda$ EMBL3 DNA, Southern blotting and subcloning 
of hybridizing fragments from bacteriophages into pMTL21 or pUC19 were performed using standard protocols (Sambrook et al., 1989). DNA sequencing was performed by Microsynth. T. rubrum and Arth. benhamiae cDNAs were obtained by RT-PCR using homologous primers designed from genomic DNA sequences.

RNA preparations and Northern-blotting experiments. The RNA of filamentous fungi was prepared from 10-day-old $100 \mathrm{ml}$ liquid cultures in different media. The mycelium was ground in liquid nitrogen to a fine powder using a mortar and pestle, and the total RNA was isolated using an RNeasy Plant Mini Kit (Qiagen). Northern-blotting experiments were performed as described by Sambrook et al. (1989).

\section{RESULTS}

\section{Sulphite secreted by filamentous fungi}

T. rubrum and Arth. benhamiae grew well at $30^{\circ} \mathrm{C}$ in a medium containing $0.2 \%$ arginine and $0.07 \%$ cystine as the only nitrogen sources. In contrast, germ tubes alone developed from $A$. fumigatus conidia in cystine-arginine liquid medium. After 20 days growth, a substantial amount of $S$-sulphocysteine was measured in the culture supernatants of the dermatophytes $\left(0.7-2.0 \mu \mathrm{mol} \mathrm{ml}^{-1}\right)$. Concomitantly, sulphite was detected at concentrations of $0-0.16 \mu \mathrm{mol} \mathrm{ml} l^{-1}$ and $0.64-0.80 \mu \mathrm{mol} \mathrm{ml} l^{-1}$ for T. rubrum and Arth. benhamiae, respectively. S-Sulphocysteine was also detected in the supernatants of the dermatophyte and $A$. fumigatus cultures in KSP protein medium at concentrations ranging from 0.01 to $0.035 \mu \mathrm{mol} \mathrm{ml}{ }^{-1}$, at which point substantial endoproteolytic activity was recorded and the media were totally clarified (15-20 days for dermatophytes, 30-40 days for A. fumigatus). Free sulphite was not detected in KSP protein medium. The MIC for sulphite measured in YEPD + TA medium was $3 \mathrm{mM}$ for the dermatophytes and $1.5 \mathrm{mM}$ for $A$. fumigatus.

\section{Cloning of genes encoding $\boldsymbol{A}$. fumigatus and dermatophyte sulphite efflux pumps}

The high concentrations of $S$-sulphocysteine and sulphite in dermatophyte culture supernatants in cystine media led us to identify sulphite efflux pumps in these fungi. A sulphite efflux pump, Ssul (called in this paper SceSsulp), has been characterized elsewhere in S. cerevisiae (Avram \& Bakalinsky, 1997). Overexpression of the gene SceSSU1 enhances the resistance of $S$. cerevisiae to sulphite, which is used as a preservative agent in fermentations (Park et al., 1999; Park \& Bakalinsky, 2000; Donalies \& Stahl, 2002). SceSsulp belongs to the TDT family, which includes the $E$. coli tellurite transporter and the Schizosaccharomyces pombe malate transporter encoded by the genes TEHA and MAEI, respectively (Walter et al., 1991; Grobler et al., 1995; see the TransportDB database, http://www.membranetransport. org/). Our strategy to find sulphite efflux pumps in dermatophytes consisted of trying to isolate the SceSSU1 orthologue in A. fumigatus for which the genome sequence is available. If a hit was obtained, the orthologues in dermatophyte genomes could subsequently be sought. Indeed, it is known that the nucleotide sequences of dermatophyte genes exhibit $50-70 \%$ identity to the orthologous genes of Aspergillus spp. (Jousson et al., 2004a, b; Monod et al., 2005).

A BLASTP analysis with SceSsulp on the A. fumigatus Af293 genome (www.tigr.org/tdb/e2k1/aful) revealed seven genes encoding putative transporters of the TDT family. Three transporters with the highest similarity to SceSsulp, corresponding to Afu7g01790, Afu3g14640 and Afu1g13360 in the TransportDB database, were retained for gene expression experiments in S. cerevisiae (Fig. 2). Three corresponding cDNAs could specifically be amplified using $5^{\prime}$-sense and $3^{\prime}$-antisense primers (P1-P6, Table 1) by RT-PCR of $A$. fumigatus D141 RNA, or by PCR based on DNA extraction from a pool of $10^{6}$ clones of a previously

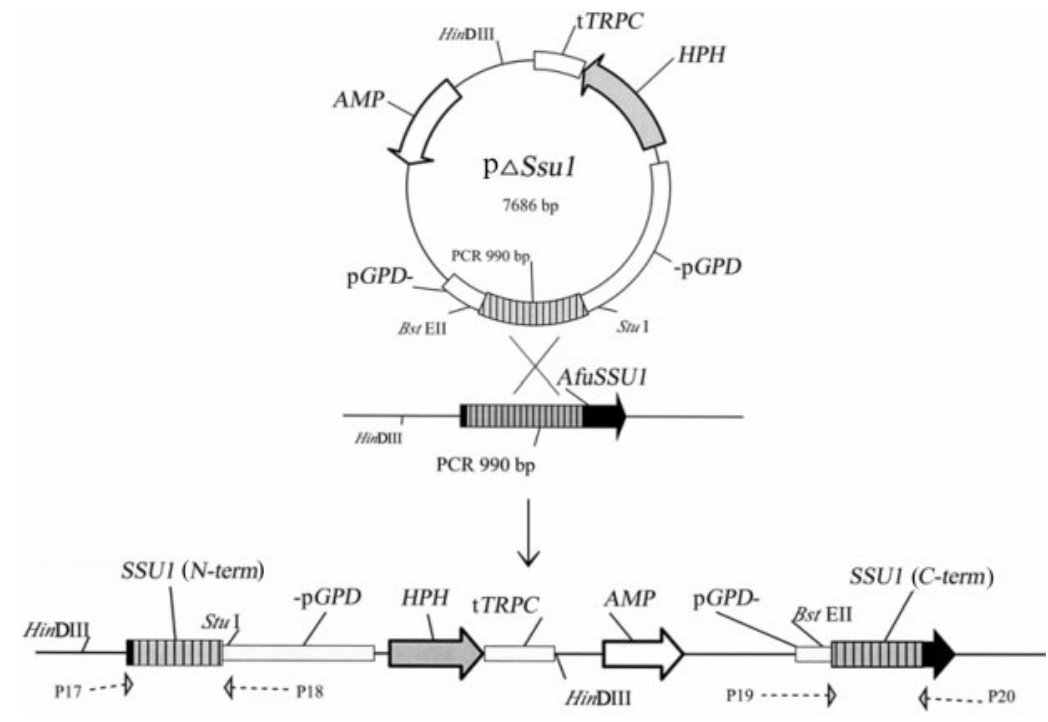

Fig. 1. Plasmid construct and predicted outcome of the $\mathrm{p} \Delta S$ su1 integration event. A $990 \mathrm{bp}$ internal PCR fragment of AfuSSU1 was cloned into the pAN7.1 plasmid (Punt et al., 1987). pAN7.1 carries the hygromycin resistance gene $(H P H)$ from $E$. coli as a dominant selectable marker under the control of the GPD promoter ( $p G P D$ ) and TRPC terminator (tTRPC) from $A$. nidulans. Homologous recombination of the plasmid construct with AfuSSU1 by a single crossover event resulted in the generation of two incomplete copies of the A. fumigatus gene [labelled SSU1 ( $N$ term) and SSU1 (C-term)] separated by the linearized sequence of pAN7.1. The primers used to screen for the incomplete AfuSSU1 gene were P17 and P18 (5' fragment) and P19 and P20 ( $3^{\prime}$ fragment). Further information on primers is in Table 1. 


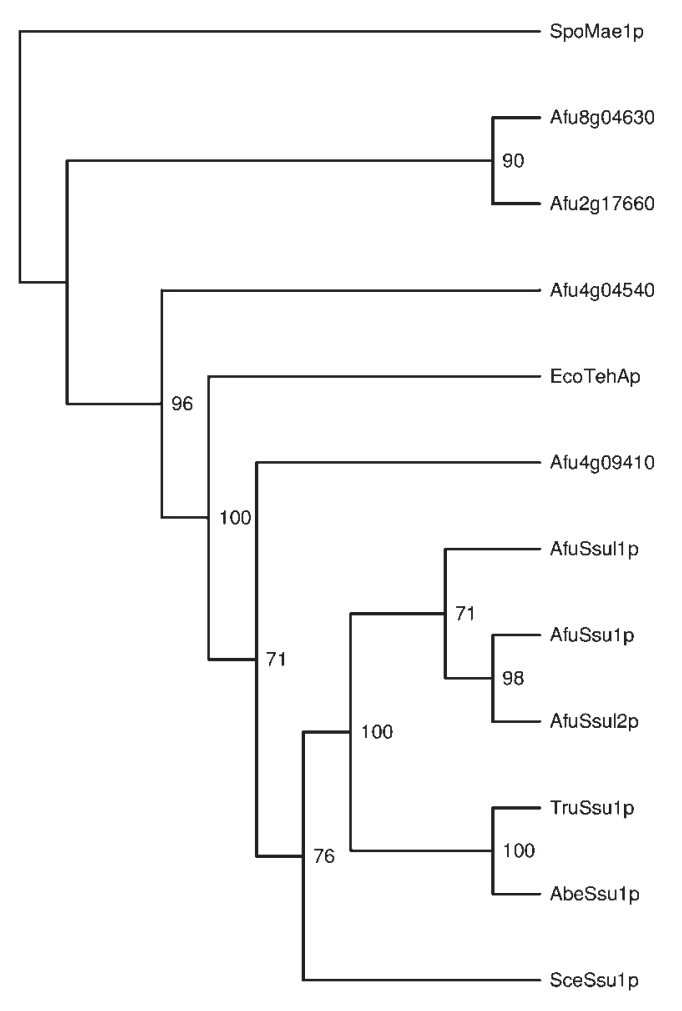

Fig. 2. Neighbour-joining (NJ) phylogenetic tree of TDT transporters, including seven members recorded in $A$. fumigatus. AfuSsu1p, AfuSsul1p and AfuSsul2p substitute Afu7g01790, Afu3g14640 and Afu1g13360, respectively. The deduced amino acid sequences of AfuSsu1p, AfuSsul1p and AfuSsul2p revealed some differences from the deposited amino acid sequences for Afu3g14640, Afu1g13360 and Afu7g01790 in the TransportDB database. The branch lengths are proportional to the similarity between amino acid sequences. Amino acid sequence accession numbers at NCBI are as follows: SpoMae1, AAC49149; SceSsu1p, NP_015233; TruSsu1p, DQ777768; AbeSsu1p, EF035480; AfuSsu1p, AAX54670; AfuSsul1p, AAX54671; AfuSsul2p, AAX54672; Afu8g04630, EAL85231; Afu4g04540, EAL84602; Afu4g09410, EAL89846; Afu2g17660, EAL94063; E. coli TehAp (EcoTehAp), AAA19563.

constructed cDNA library as the template. The nucleotide sequences of the obtained cDNA fragments were $100 \%$ identical to sequences in the A. fumigatus Af293 genome from which introns were removed. However, their deduced amino acid sequences revealed some differences from the deposited amino acid sequences of Afu7g01790, Afu3g14640 and Afulg13360 because of differences in intron positions. The three A. fumigatus cDNAs were cloned into the $S$. cerevisiae expression vector pYES2 under control of the GAL promoter, and the resulting plasmids were transformed into S. cerevisiae. Only transformants encoding one of the three transporter genes, corresponding to Afu7g01790, showed increased sulphite resistance on galactose media (Fig. 3). This gene was called AfuSSU1 (GenBank accession no.
(A)

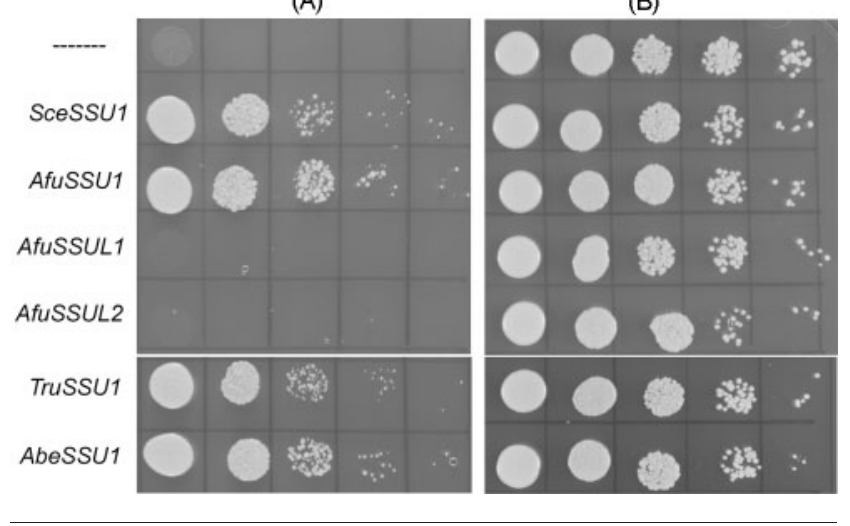

Fig. 3. (A) Susceptibility and resistance to sulphite $(1.6 \mathrm{mM})$ of $S$. cerevisiae transformed with pYES2 (top line) and with different plasmids encoding TDT transporters. S. cerevisiae was spotted at different dilutions on YEPG +TA plates, as described in Methods. (B) As a control, S. cerevisiae was spotted on plates without sulphite. The plates were incubated at $30^{\circ} \mathrm{C}$ for 2 days.

AY861352). The two other genes were called AfuSSUL1 and AfuSSUL2, encoding Ssu-like protein 1 and Ssu-like protein 2 in A. fumigatus (GenBank accession nos AY861353 and AY861354, respectively). AfuSSU1, AfuSSUL1 and AfuSSUL2 revealed similar collinear structures with four introns and five exons.

In parallel, A. fumigatus disruption mutants were constructed and tested for sulphite resistance, as described in Methods. A. fumigatus ssul mutants were highly sensitive to sulphite (Fig. 4), while the resistance to sulphite of ssull and ssul2 mutants was comparable to that of the wild-type strain of the fungus. These results confirmed that only AfuSSU1

\section{wt ssu1 ssul1 ssul2}

(A)

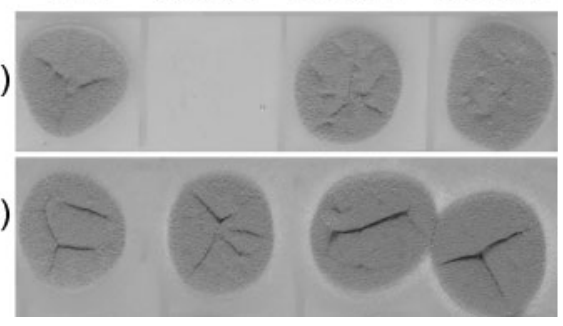

Fig. 4. Susceptibility and resistance to sulphite of $A$. fumigatus D141 wild-type (wt) and mutated strains. YEPD+TA plates with $0.8 \mathrm{mM}$ sulphite $(\mathrm{A})$ and without sulphite $(\mathrm{B})$ were spotted with $5 \mu \mathrm{l}$ of a suspension of $A$. fumigatus conidia $\left(10^{6} \mathrm{ml}^{-1}\right)$. The plates were incubated at $30^{\circ} \mathrm{C}$ for 2 days. YEPD +TA plates containing sulphite were prepared by spreading an appropriate amount of freshly made $\mathrm{Na}_{2} \mathrm{SO}_{3}$ stock solution onto agar plates containing $25 \mathrm{ml}$ medium, and allowing the sulphite to dry and diffuse overnight at room temperature. 
encodes a sulphite efflux pump among the three selected genes of A. fumigatus encoding transporters of the TDT family. However, the A. fumigatus ssu1 mutants grew in a similar manner to the wild-type strain in SP and KSP media, and $S$-sulphocysteine was detected in the culture supernatant in KSP medium.

AfuSSU1, AfuSSUL1 and AfuSSUL2 were predicted to encode proteins of 396, 396 and 436 amino acid residues, with molecular masses of approximately 43.6, 44.0 and $47.6 \mathrm{kDa}$, respectively. Like the Schiz. pombe malate transporter encoded by the MAEI gene (Grobler et al., 1995), the deduced amino acid sequences revealed proteins with hydrophilic $\mathrm{N}$ and $\mathrm{C}$ termini and 10 putative membrane-spanning helices which are typical of membrane transport proteins (Fig. 5). The length of the hydrophilic linkers ranged from 3 to 20 amino acids.

A $1200 \mathrm{bp}$ fragment containing the whole nucleotide sequence of AfuSSU1 was used as a probe for screening a $\lambda$ EMBL3 phage T. rubrum genomic DNA library. All isolated hybridizing clones contained a nucleotide sequence encoding a gene $50 \%$ identical to AfuSSU1. The corresponding cDNA encoding a putative sulphite transporter could be specifically amplified by RT-PCR using $5^{\prime}$-sense and $3^{\prime}$ antisense primers (Table 1) and total RNA extracted from $T$. rubrum mycelium in cystine-arginine medium. An Arth. benhamiae cDNA encoding a putative transporter differing from that of T. rubrum by two amino acids (Val63 and Ala206 in Arth. benhamiae instead of Ile63 and Val206 in T. rubrum) was also amplified using the same primers. $S$. cerevisiae became resistant to $1.6 \mathrm{mM}$ sulphite when these amplified cDNAs were expressed under the control of the GAL promoter (Fig. 3). The genes encoding these sulphite transporters in T. rubrum and Arth. benhamiae were called TruSSU1 and AbeSSU1 (GenBank accession nos DQ777768 and EF035480, respectively). TruSSU1 and AbeSSU1 revealed an intron-exon structure similar to that of AfuSSU1. Both TruSSU1 and AbeSSU1 were predicted to encode proteins of 375 amino acid residues with molecular masses of approximately $42 \mathrm{kDa}$, hydrophilic $\mathrm{N}$ and $\mathrm{C}$ termini, and 10 putative membrane-spanning helices (Fig. 5).

\section{Expression of sulphite transporter genes in $A$. fumigatus and dermatophytes}

Northern-blot analysis revealed that TruSSU1 and AbeSSU1 encode single transcripts of approximately $2000 \mathrm{bp}$ each. Expression of TruSSU1 and AbeSSU1 in cystine-arginine and KSP media was higher than that in Sabouraud and SP media (Fig. 6). Although AfuSSU1 DNA could be amplified by RT-PCR with RNA extracted from $A$. fumigatus grown in YEPD + TA containing $1.2 \mathrm{mM}$ sulphite $(300 \mathrm{mM}$ below the MIC), SP and KSP media, no AfuSSU1 signal was detected in comparably sensitive Northern blotting experiments and utilizing the whole AfuSSU1 cDNA as a probe.

\section{DISCUSSION}

We present what is believed to be the first molecular genetic report on sulphite efflux pumps in dermatophytes and in filamentous fungi. The number of genes within the fungal genome that encode transporters of the TDT family to which these pumps belong varies from species to species. In $S$. cerevisiae. SceSsulp is the only member of this family. In contrast, the TDT family has seven, six, three and two members in A. fumigatus, Aspergillus oryzae, A. nidulans and Neurospora crassa, respectively (see the TransportDB

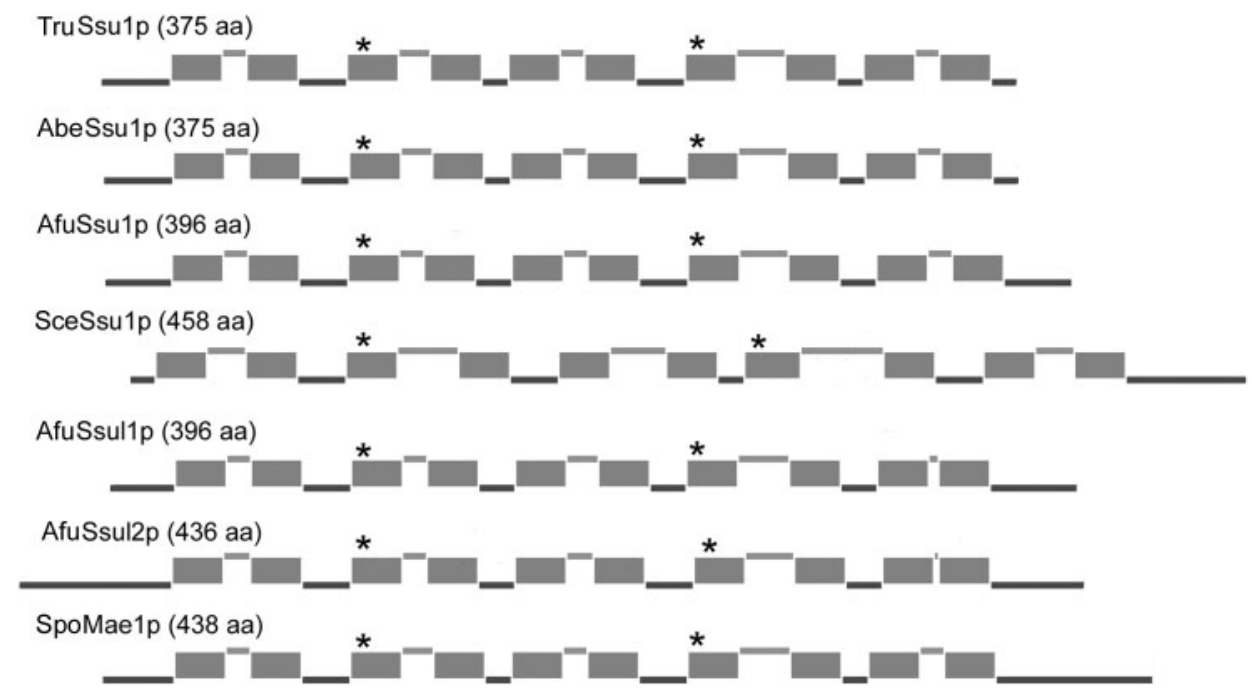

Fig. 5. Transmembrane protein topology predicted using PRODIV-TMHMM (http://www.pdc.kth.se/ hakanv/prodiv-tmhmm/) (Viklund \& Elofsson, 2004). The plot was obtained by calculating the total probability that a residue sits within a helix (box), inside (lowered line) or outside the protein (raised line), summed over all possible paths through the model. The two portions of the alignment shown in Fig. 7 are indicated by asterisks. 


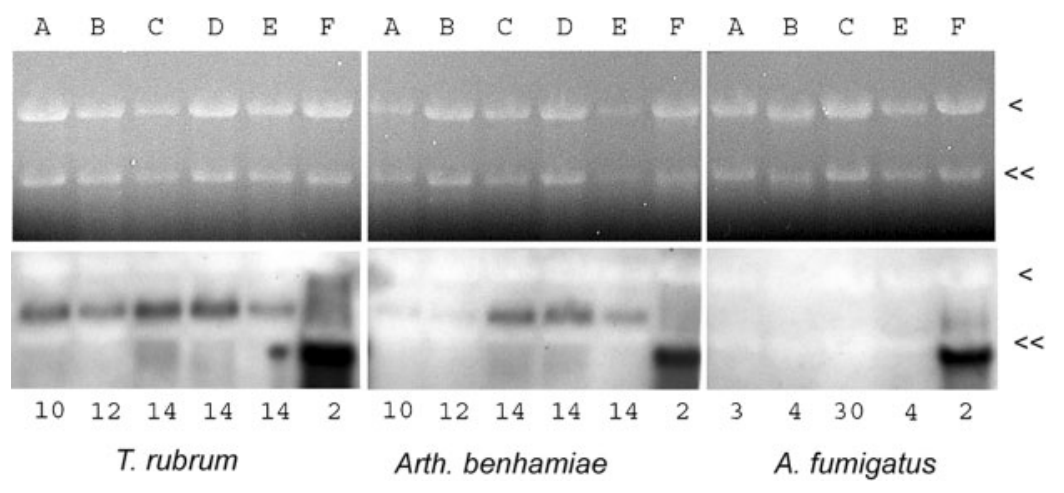

Fig. 6. Northern blots of T. rubrum, Arth. benhamiae and $A$. fumigatus total RNA hybridized with cDNA fragments (pYES2 inserts) encoding the whole of TruSsu1p, AbeSsu1p and AfuSsu1p, respectively, as a probe. Mycelium was harvested from growing hyphae in the following liquid media: Sabouraud (A), SP (B), KSP (C), cystine-arginine (D) and YEPD + TA containing $1.2 \mathrm{mM}$ sulphite (E). The variable number of days of each culture is indicated in the bottom line of the figure. In SP and KSP media, substantial endoproteolytic activity was recorded, and the media were totally clarified. In cystine-arginine medium, a substantial amount of $S$-sulphocysteine was measured in the culture supernatants. Approximately $4 \mu \mathrm{g}$ total RNA was loaded in each lane. As an intensity standard, $4 \mu \mathrm{g}$ total RNA from $S$. cerevisiae grown on synthetic minimal medium with galactose and expressing TruSSU1, AbeSSU1 and AfuSSU1 was loaded in parallel (lanes F). The position of the hybridizing bands obtained from $S$. cerevisiae transformants is lower, because only the parts of the cDNAs encoding TruSSUp, AbeSSU1p and AfuSSU1p, respectively, were cloned in pYES2. $28 S$ and $18 \mathrm{~S}$ rRNAs are indicated to the right of the figure by $<$ and $\ll$, respectively.

database). However, gene disruption of AfuSSU1 and of the two more closely related paralogues in A. fumigatus revealed that only AfuSSU1 encodes a sulphite efflux pump. The multitude of transporters of the TDT family in a saprophytic and opportunistic fungus such as A. fumigatus is likely to confer other selective advantages in terms of resistance to the different chemical compounds which are present in varied environments. TruSsulp and AbeSsulp are believed to be the first transporters of the TDT family to be described in dermatophytes. No further paralogues were identified from a screening of the T. rubrum genomic library performed with TruSSU1 as probe.

AfuSsulp, TruSsulp and AbeSsulp are only $20 \%$ identical to SceSsulp. In contrast, A. fumigatus transporters of the TDT family which are not sulphite efflux pumps are more similar to AfuSsulp (Fig. 2). Alignments of TDT transporters included in Fig. 2 show that the identified sulphite efflux pumps have only two short conserved amino acid sequences (Fig. 7). Two potential signature sequences for sulphite transporters in the TDT family could be derived from the portions of the alignment shown in Fig. 7 as follows:

(1) GT(F/Y)PMG(F/L)XTIIN

\section{(2) $\mathrm{LP}(\mathrm{I} / \mathrm{L}) \mathrm{GP}(\mathrm{L} / \mathrm{M}) \mathrm{GQG}(\mathrm{G} / \mathrm{S})(\mathrm{F} / \mathrm{Y}) \mathrm{G}$}

Sulphite is a product of the metabolism of cysteine compounds assimilated by fungi. Although a regular fungal metabolite, sulphite is potentially toxic in the cytoplasm (Thomas \& Surdin-Kerjan, 1997). Excess sulphite is generally excreted by fungi after oxidation in the form of inorganic sulphate (Obata \& Ishikawa, 1959; Kunert, 1989).

\begin{tabular}{|c|c|c|c|c|c|c|c|c|c|c|c|c|c|}
\hline TruSsulp & G & $\mathrm{I}$ & F & $\mathrm{P}$ & M & G & F & A & 1 & - & 1 & $\mathbb{N}$ & $104-115$ \\
\hline Abessulp & - & . & - & . & - & - & 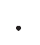 & & - & . & 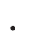 & - & $104-115$ \\
\hline Afussulp & - & 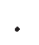 & - & . & - & • & $\mathrm{L}$ & $\mathrm{T}$ & . & . & . & . & $103-114$ \\
\hline SceSsulp & . & . & $\mathrm{Y}$ & . & . & . & $\mathrm{L}$ & $\mathrm{V}$ & $\bullet^{\circ}$ & . & 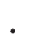 & • & $94-105$ \\
\hline Afussullp & A & . & $\mathrm{C}$ & . & - & . & 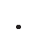 & - & . & $\mathrm{L}$ & 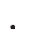 & $E$ & $.02-113$ \\
\hline Afussul2p & $S$ & . & . & . & . & . & L & 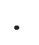 & 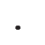 & 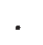 & V & & $39-150$ \\
\hline SpoMae1p & $\mathrm{P}$ & 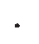 & A & L & $\mathrm{L}$ & A & I & . & . & $S$ & 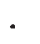 & S & $5-116$ \\
\hline Trussulp & $\mathrm{L}$ & F & I & G & $\mathrm{P}$ & $\mathrm{L}$ & G & $Q$ & G & $\mathrm{G}$ & F & $\mathrm{G}$ & 53 \\
\hline AbeSsulp & - & & - & 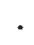 & - & . & . & . & . & - & . & 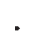 & $42-253$ \\
\hline Afussulp & . & 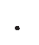 & L & 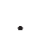 & ${ }^{\circ}$ & 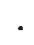 & . & ${ }^{\circ}$ & . & $\cdot$ & Y & & $242-253$ \\
\hline scessulp & . & L & $\mathrm{L}$ & . & . & M & . & 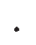 & . & $S$ & 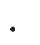 & & $257-268$ \\
\hline Afussul1p & . & & L & & & . & . & F & . & & & $\cdot$ & $241-252$ \\
\hline Afussul2p & $\cdot$ & ${ }^{\circ}$ & M & ${ }^{\circ}$ & . & 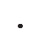 & • & S & . & $\mathrm{T}$ & • & A & $278-289$ \\
\hline Spolaelp & $I$ & I & V & S & & $\mathrm{P}$ & $S$ & F & $\mathrm{T}$ & & $\mathrm{L}$ & $\mathrm{T}$ & $242-253$ \\
\hline
\end{tabular}

Fig. 7. Domains of homology between the deduced amino acid sequences of suphite efflux pumps TruSsu1p, AbeSsu1p, AfuSsu1p and SceSsu1p, two other closely related members of the TDT family, AfuSsul1p and AfuSsul1p, and SpoMae1p (amino acid sequence accession numbers at NCBI in legend of Fig. 2). Numbers on the right indicate the amino acid positions in the aligned proteins. Dots represent identical amino acids. 
Excretion of sulphite mediated by an efflux pump represents an alternative detoxification pathway for dermatophytes during infection of the epidermal stratum corneum, hair and nails, which are rich in cysteine. At the same time, the ability of dermatophytes to grow within hard keratin depends on the secretion of sulphite in order to reduce proteins. Once disulphide bridges are directly cleaved by sulphitolysis, reduced proteins become accessible to further digestion by various secreted endo- and exoproteases. Therefore, efflux-pump-mediated sulphite detoxification and sulphitolysis may be considered as complementary functions in the digestion of compact keratinous tissue.

The relatively high expression of TruSSU1 and AbeSSU1 compared to that of AfuSSU1 (Fig. 6) likely reflects a property of dermatophytes which renders these fungi pathogenic in the epidermal stratum corneum, hair and nails. Dermatophytes were able to grow more rapidly than A. fumigatus in KSP medium. Hard keratin grains were totally digested after 20 days under our culture conditions, while 40 days were necessary to observe clarification of the medium with $A$. fumigatus. Although sometimes isolated as a contaminant from nails, the latter fungus is not an aetiologic agent of infection in hard keratinous tissues. Apparently, A. fumigatus growth in keratin-containing medium does not depend on AfuSsul activity, since the A. fumigatus ssu1 mutants were able to grow well in KSP medium, and $S$-sulphocysteine was detected in the culture supernatant. Thus, the existence of another sulphite efflux pump cannot be excluded in A. fumigatus, but it is also possible that small amounts of sulphite can leave the mycelium by a route other than that of sulphite transporters.

No fewer than 16 genes encoding secreted endo- and exopeptidases have been found in dermatophytes (Jousson et al., 2004a, b; Monod et al., 2005). The hypothesis that one keratinase, alone or with further proteases, decomposes hard keratin, has now been abandoned (Kunert, 1992). Dermatophyte-secreted subtilisins have been shown to be at most $50 \%$ more active towards keratin azure than proteinase $\mathrm{K}$ or subtilisin Carlsberg, with the last two enzymes both in the presence of $\beta$-mercaptoethanol (Jousson et al., 2004b). Sulphitolysis is so far the sole known dermatophyte mechanism that allows the reduction of disulphide bridges, a bottleneck in the process of degradation of compact keratinized tissues. In contrast, the activities of the dermatophyte-secreted proteases are redundant for the digestion of reduced protein (Jousson et al., 2004b; Monod et al., 2005). Therefore, sulphite transporters, rather than secreted proteases, could be a target of choice to treat dermatophyte infection. Given the absence of TDT transporters in humans, mechanism-based toxicity would most likely be minimal.

\section{ACKNOWLEDGEMENTS}

We thank Dr Kunlin Zhang for bioinformatic help, and Dr Bertrand Favre, Dr Lee Laurent-Applegate and Dr Massimo Lurati for critical review of the manuscript and assistance with the English. This work was supported by the Swiss National Foundation for Scientific Research, grant 3100-105313/1.

\section{REFERENCES}

Avram, D. \& Bakalinsky, A. T. (1997). SSU1 encodes a plasma membrane protein with a central role in a network of proteins conferring sulfite tolerance in Saccharomyces cerevisiae. J Bacteriol 179, 5971-5974.

Chambers, S. P., Prior, S. E., Barstow, D. A. \& Minton, N. P. (1988), The pMTL nic ${ }^{-}$cloning vectors. Improved pUC polylinker regions to facilitate the use of sonicated DNA for nucleotide sequencing. Gene 68, 139-149.

Cove, D. J. (1966). The induction and repression of nitrate reductase in the fungus Aspergillus nidulans. Biochim Biophys Acta 113, 51-56.

Denikus, N., Orfaniotou, F., Wulf, G., Lehmann, P. F., Monod, M. \& Reichard, U. (2005). Fungal antigens expressed during invasive aspergillosis. Infect Immun 73, 4704-4713.

Donalies, U. E. \& Stahl, U. (2002). Increasing sulphite formation in Saccharomyces cerevisiae by overexpression of MET14 and SSU1. Yeast 19, 475-484.

Fumeaux, J., Mock, M., Ninet, B., Jan, I., Bontems, O., Léchenne, B., Lew, D., Panizzon, R. G., Jousson, O. \& Monod, M. (2004). First report of Arthroderma benhamiae in Switzerland. Dermatology 208, 244-250.

Grobler, J., Bauer, F., Subden, R. E. \& vanVuuren, H. J. J. (1995). The mael gene of Schizosaccharomyces pombe encodes a permease for malate and other C4 dicarboxylic acids. Yeast 11, 1485-1491.

Grossberger, D. (1987). Minipreps of DNA from bacteriophage lambda. Nucleic Acids Res 15, 6737.

Henderson, J. W., Ricker, R. D., Bidlingmeyer, B. A. \& Woodward, C. (2000). Rapid, Accurate, Sensitive and Reproducible Analysis of Amino Acids. Agilent technical note no. 5980-1193E.

Jousson, O., Léchenne, B., Bontems, O., Capoccia, S., Mignon, B., Barblan, J., Quadroni, M. \& Monod, M. (2004a). Multiplication of an ancestral gene encoding secreted fungalysin preceded species differentiation in the dermatophytes Trichophyton and Microsporum. Microbiology 150, 301-310.

Jousson, O., Léchenne, B., Bontems, O., Mignon, B., Reichard, U., Barblan, J., Quadroni, M. \& Monod, M. (2004b). Secreted subtilisin gene family in Trichophyton rubrum. Gene 339, 79-88.

Kunert, J. (1972a). Keratin decomposition by dermatophytes: evidence of the sulphitolysis of the protein. Experientia 28, 1025-1026.

Kunert, J. (1972b). Thiosulphate esters in keratin attacked by dermatophytes in vitro. Sabouraudia 10, 6-13.

Kunert, J. (1975). Formation of sulphate, sulphite and $S$-sulphocysteine by the fungus Microsporum gypseum during growth on cystine. Folia Microbiol 20, 142-151.

Kunert, J. (1976). Keratin decomposition by dermatophytes. II. Presence of $S$-sulfocysteine and cysteic acid in soluble decomposition products. Z Allg Mikrobiol 16, 97-105.

Kunert, J. (1982). Utilization of L- and DL-cystine by the fungus Microsporum gypseum. Folia Microbiol 27, 390-394.

Kunert, J. (1989). Utilization of L-cystine as a source of carbon and nitrogen by various fungi. Acta Univ Palacki Olomuc Fac Med 123, 351-364.

Kunert, J. (1992). Effect of reducing agents on proteolytic and keratinolytic activity of enzymes of Microsporum gypseum. Mycoses 35, 343-348. 
Kunert, J. (2000). Physiology of keratinophilic fungi. Rev Iberoam Micol 17, 77-85.

Kwong-Chung, K. J. \& Bennet, J. E. (1992). Medical Mycology. Philadelphia and London: Lea and Febiger.

Monod, M., Togni, G., Hube, B. \& Sanglard, D. (1994). Multiplicity of genes encoding secreted aspartic proteinases in Candida species. Mol Microbiol 13, 357-368.

Monod, M., Léchenne, B., Jousson, O., Grand, D., Zaugg, C., Stocklin, R. \& Grouzmann, E. (2005). Aminopeptidases and dipeptidyl-peptidases secreted by the dermatophyte Trichophyton rubrum. Microbiology 151, 145-155.

Obata, Y. \& Ishikawa, Y. (1959). Biochemical studies on sulfurcontaining aminoacids. II. Sulfate formation from L-cystine by molds. J Biochem 46, 293-295.

Paris, S. (1994). Isolation of protease negative mutants of Aspergillus fumigatus by insertion of a disrupted gene. In Molecular Biology of Pathogenic Fungi: a Laboratory Manual, pp. 49-55. Edited by B. Maresca \& G. S. Kobayashi. New York: Telos Press.

Park, H. \& Bakalinsky, A. T. (2000). SSU1 mediates sulphite efflux in Saccharomyces cerevisiae. Yeast 16, 881-888.

Park, H., Lopez, N. I. \& Bakalinsky, A. T. (1999). Use of sulfite resistance in Saccharomyces cerevisiae as a dominant selectable marker. Curr Genet 36, 339-344.

Punt, S. J., Oliver, P. O., Dingemanse, M. A., Pouwels, P. H. \& van den Hondel, C. A. M. J. J. (1987). Transformation of Aspergillus based on hygromycin B resistance marker from Escherichia coli. Gene 56, $117-124$.
Ruffin, P., Andrieu, S., Biserte, G. \& Biguet, J. (1976). Sulphitolysis in keratinolysis. Biochemical proof. Sabouraudia 14, 181-184.

Sambrook, J., Fritsch, E. F. \& Maniatis, T. (1989). Molecular Cloning: a Laboratory Manual, 2nd edn. Cold Spring Harbor, NY: Cold Spring Harbor Laboratory.

Scaringelli, F. P., Saltzman, B. E. \& Frey, S. A. (1967). Spectrophotometric determination of atmospheric sulfur dioxide. Anal Chem 39, 1709-1726.

Sherman, F. (1991). Mapping yeast genes. Methods Enzymol 194, 38-57.

Thomas, D. \& Surdin-Kerjan, Y. (1997). Metabolism of sulfur amino acids in Saccharomyces cerevisiae. Microbiol Mol Biol Rev 61, 503-532.

Tilburn, J., Scazzocchio, C., Taylor, G. G., Zabicky-Zissman, J. H., Lockington, R. A. \& Davies, R. W. (1983). Transformation by integration in Aspergillus nidulans. Gene 26, 205-221.

Viklund, H. \& Elofsson, A. (2004). Best alpha-helical transmembrane protein topology predictions are achieved using hidden Markov models and evolutionary information. Protein Sci 13, 1908-1917.

Walter, E. G., Weiner, J. H. \& Taylor, D. E. (1991). Nucleotide sequence and overexpression of the tellurite-resistance determinant from the IncHII plasmid pHH1508a. Gene 101, 1-7.

Weitzman, I. \& Summerbell, R. C. (1995). The dermatophytes. Clin Microbiol Rev 8, 240-259.

Edited by: N. L. Glass 